\title{
On a degenerate q-Euler polynomials and numbers with weight
}

Guhyun Na, Yunju Cho, Jin-Woo Park*

Department of Mathematics Education, Daegu University, 38453, Republic of Korea.

\begin{abstract}
In this paper, we define the $p$-adic q-integral on $\mathbb{Z}_{p}$ with weight which is a generalization of Kim's definition in [T. Kim, Russ. J. Math. Phys., 9 (2002), 288-299], and derive some new and interesting identities related to degenerate q-Euler polynomials with weight and some special functions.
\end{abstract}

Keywords: p-Adic q-integral with weight, degenerate q-Euler polynomials, q-Euler polynomials with weight.

2010 MSC: 33E20, 05A30, 11B65, 11505.

(C)2020 All rights reserved.

\section{Introduction}

For a long time, special functions have been considered the particular province of pure and applied mathematics, and many special functions have been appeared as solutions of differential equations or integrals of elementary functions (see [2]). In number theory, various special functions have been studied, such as Dirichlet series, Bernoulli polynomials, Euler polynomials, Genocchi polynomials, Bell polynomials, the Stirling numbers of the first and second kinds and harmonic numbers, Daehee polynomials and Chaghee polynomials. These results shows how to apply special functions and polynomials to the mathematics and mathematical physics (see [1-4, 6-10, 12-29]). In particular, Cesarano presented some techniques regarding the generating functions used in [7], and Marin showed these identities can be applicable to the theory of porous materials (see [24]).

From now on, we introduce some definitions and notations which are useful tools in this paper.

For a given odd prime number $p, \mathbb{Z}_{p}, \mathbb{Q}_{p}$, and $\mathbb{C}_{p}$ denote the ring of $p$-adic integers, the field of p-adic rational numbers, and the completions of algebraic closure of $\mathbb{Q}_{p}$, respectively. The $p$-adic norm is normalized as $|p|_{p}=\frac{1}{p}$.

Let $\mathrm{q} \in \mathbb{C}_{\mathrm{p}}$ be an indeterminate with $|\mathrm{q}-1|_{\mathrm{p}}<\mathrm{p}^{-\frac{1}{\mathrm{p}-1}}$. Then the $\mathrm{q}$-analogue of number $\mathrm{x}$ is defined as $[x]_{\mathrm{q}}=\frac{1-\mathrm{q}^{\mathrm{x}}}{1-\mathrm{q}}$. Note that $\lim _{\mathrm{q} \rightarrow 1}[x]_{\mathrm{q}}=x$ for each $x \in \mathbb{Z}_{\mathrm{p}}$.

\footnotetext{
*Corresponding author

Email addresses: naguhyun@naver.com (Guhyun Na), coj9804@naver .com (Yunju Cho), a0417001@knu.ac.kr (Jin-Woo Park)

doi: $10.22436 /$ jmcs.020.03.04
}

Received: 2019-08-12 Revised: 2019-11-05 Accepted: 2019-11-13 
Let $C\left(\mathbb{Z}_{p}\right)$ be the set of all continuous function on $\mathbb{Z}_{p}$. The fermionic $p$-adic $q$-integral of $f \in C\left(\mathbb{Z}_{p}\right)$ is defined by Kim as

$$
\begin{aligned}
I_{-q}(f) & =\int_{\mathbb{Z}_{p}} f(x) d \mu_{-q}(x)=\lim _{N \rightarrow \infty} \frac{1}{\left[p^{N}\right]_{-q}} \sum_{x=0}^{p^{N}-1} f(x)(-q)^{x} \\
& =\lim _{N \rightarrow \infty} \frac{[2]_{q}}{2} \sum_{x=0}^{p^{N}-1} f(x)(-q)^{x},(\text { see }[11,12]) .
\end{aligned}
$$

As is well-known that the $n^{\text {th }}$ Euler polynomials $E_{n}(x)$ is defined by the Appell sequence with $g(x)=$ $\frac{1}{2}\left(e^{t}+1\right)$, giving the generating function

$$
\sum_{n=0}^{\infty} E_{n}(x) \frac{t^{n}}{n !}=\frac{2}{e^{t}+1} e^{x t},(\text { see }[6,10])
$$

By using the fermionic $\mathrm{p}$-adic q-integral, the q-Euler polynomials are defined by the generating function to be

$$
\sum_{n=0}^{\infty} E_{n, q}(x) \frac{t^{n}}{n !}=\int_{\mathbb{Z}_{p}} e^{(x+y) t} d \mu_{-q}(y)=\frac{[2]_{q}}{q e^{t}+1} e^{x t},(\text { see }[15-17,26-28]),
$$

and, by the Maclaurin expansion of $e^{(x+y) t}$, we can obtain the $n^{\text {th }} q-$ Euler polynomial $E_{n, q}(x)$ as

$$
E_{n, q}(x)=\int_{\mathbb{Z}_{p}}(x+y)^{n} d \mu_{-q}(y),(n \geqslant 0)(\text { see }[4,5,16,17,28,29]) .
$$

In [6], Carlitz introduced the degenerate polynomials and numbers which are related to q-Euler polynomials as follows

$$
\sum_{n=0}^{\infty} E_{n}(x \mid \lambda) \frac{t^{n}}{n !}=\left(\frac{2}{(1+\lambda t)^{\frac{1}{\lambda}}+1}\right)^{r}(1+\lambda t)^{\frac{x}{\lambda}}
$$

where $\lambda \in \mathbb{R}$. Note that, by (1.3), we know that

$$
\lim _{\lambda \rightarrow 0} \sum_{n=0}^{\infty} E_{n}(x \mid \lambda) \frac{t^{n}}{n !}=\lim _{\lambda \rightarrow 0}\left(\frac{2}{(1+\lambda t)^{\frac{1}{\lambda}}+1}\right)^{r}(1+\lambda t)^{\frac{x}{\lambda}}=\left(\frac{2}{e^{t}+1}\right)^{r} e^{x t}=\sum_{n=0}^{\infty} E_{n}(x) \frac{t^{n}}{n !},
$$

and so

$$
\lim _{\lambda \rightarrow 0} E_{n}(x \mid \lambda)=E_{n}(x) .
$$

The Stirling numbers of the first kind are defined by

$$
(x)_{n}=x(x-1) \cdots(x-n+1)=\sum_{l=0}^{n} S_{1}(n, l) x^{l},(n \geqslant 0),
$$

and the Stirling numbers of the second kind are given by

$$
x^{n}=\sum_{l=0}^{n} S_{2}(n, l)(x)_{l}
$$

where $(x)_{0}=1$ (see $\left.[6,8,14]\right)$. From (1.4) and (1.5), we can derive the following equations

$$
\left(e^{t}-1\right)^{n}=n ! \sum_{l=n}^{\infty} S_{2}(l, n) \frac{t^{l}}{l !},
$$


and

$$
(\log (x+1))^{n}=n ! \sum_{l=n}^{\infty} S_{1}(l, n) \frac{x^{l}}{l !},(n \geqslant 0)(\text { see }[6,8,14]) .
$$

By (1.1) and (1.4), we get

$$
E_{n, \lambda, q}(x)=\lambda^{n} \sum_{l=0}^{n} S_{1}(n, l) \int_{\mathbb{Z}_{p}}\left(\frac{[x+y]_{q}}{\lambda}\right)^{l} d \mu_{-q}(y)=\sum_{l=0}^{n} \lambda^{n-l} S_{1}(n, l) E_{l, q}(x),(\text { see }[3,9,21,22]) .
$$

In [12], Kim defined the q-Volkenborn integration, and found properties of some special polynomials, especially Euler, q-analogue of Bernoulli, Genocchi, Changhee, Daehee, and Changhee-Genocchi polynomials and numbers have been investigated by many researchers by using $p$-adic q-integral on $\mathbb{Z}_{p}$ (see $[3,5,8,9,16,17,20-23,26,29])$.

In this paper, we defined the p-adic q-integral on $\mathbb{Z}_{p}$ with weight which is a generalization of Kim's $p$-adic $q$-integral on $\mathbb{Z}_{p}$, and derived some new and interesting identities related to degenerate $q$-Euler polynomials with weight, q-Euler polynomials, Stirling numbers.

\section{Degenerate q-Euler polynomials with weight}

In this section, we assume that $q \in \mathbb{C}_{p}$ with $|q|_{p}<p^{-\frac{1}{1-p}}$.

We will generalize the definition of Kim's fermionic $p$-adic q-integral on $\mathbb{Z}_{p}$ as follows.

Definition 2.1. For each parameters $\alpha$ and $\beta$,

$$
I_{q^{\alpha}, \beta}(f)=\int_{\mathbb{Z}_{p}} q^{\beta x} f(x) d \mu_{-q^{\alpha}}(x)=\lim _{N \rightarrow \infty} \frac{1}{\left[p^{N}\right]_{-q^{\alpha}}} \sum_{x=0}^{p^{N}-1} f(x)(-1)^{x} q^{(\alpha+\beta) x} .
$$

Remark 2.2. As the special case of the Definition 2.1, if we put $\alpha=1$ and $\beta=0$, then $\mathrm{I}_{\mathbf{q}^{1}, 0}=\mathrm{I}_{-\mathrm{q}}$ is Kim's definition (see [12]).

From now on, by the Definition 2.1, we will assume that $\alpha$ is a positive integer and $\beta$ is a nonnegative integer.

By the definition of $I_{q^{\alpha}, \beta}(f)$, if we put $f_{n}(x)=f(x+n)$ for each positive integer $n$, we can derive the following equations:

$$
\begin{aligned}
& -q^{\alpha+\beta} I_{q^{\alpha}, \beta}\left(f_{1}\right)=\lim _{N \rightarrow \infty} \frac{1}{\left[p^{N}\right]_{-q^{\alpha}}} \sum_{x=0}^{p^{N}-1} f(x+1)(-1)^{x+1} q^{(\alpha+\beta)(x+1)} \\
& =\lim _{N \rightarrow \infty} \frac{1}{\left[p^{N}\right]_{-q^{\alpha}}} \sum_{x=0}^{p^{N}-1} f(x)(-1)^{x} q^{(\alpha+\beta) x} \\
& +\lim _{N \rightarrow \infty} \frac{1}{\left[p^{N}\right]_{-q^{\alpha}}}\left(f\left(p^{N}\right)(-1)^{p^{N}} q^{(\alpha+\beta) p^{N}}-f(0)(-1)^{0}\right) \\
& =\mathrm{I}_{\mathbf{q}^{\alpha}, \beta}(\mathrm{f})-[2]_{\mathbf{q}^{\alpha}} \mathrm{f}(0) \text {, } \\
& q^{2(\alpha+\beta)} I_{q^{\alpha}, \beta}\left(f_{2}\right)=\lim _{N \rightarrow \infty} \frac{1}{\left[p^{N}\right]_{-q^{\alpha}}} \sum_{x=0}^{p^{N}-1} f(x+2)(-1)^{x+2} q^{(\alpha+\beta)(x+2)} \\
& =\lim _{N \rightarrow \infty} \frac{1}{\left[p^{N}\right]_{-q^{\alpha}}} \sum_{x=0}^{p^{N}-1} f(x)(-1)^{x} q^{(\alpha+\beta) x}
\end{aligned}
$$




$$
\begin{aligned}
& +\lim _{N \rightarrow \infty} \frac{1}{\left[p^{N}\right]_{-q^{\alpha}}}\left(f\left(p^{N}\right)(-1)^{p^{N}} q^{(\alpha+\beta) p^{N}}\right. \\
& \left.+f\left(p^{N}+1\right)(-1)^{p^{N}+1} q^{(\alpha+\beta)\left(p^{N}+1\right)}-f(0)+f(1) q^{\alpha+\beta}\right) \\
& =I_{q^{\alpha}, \beta}(f)+[2]_{q^{\alpha}} \sum_{l=0}^{1}(-1)^{l+1} f(l) q^{(\alpha+\beta) l},
\end{aligned}
$$

and

$$
\begin{aligned}
-q^{3(\alpha+\beta)} \mathrm{I}_{q^{\alpha}, \beta}\left(f_{3}\right)= & \lim _{N \rightarrow \infty} \frac{1}{\left[p^{N}\right]_{-q^{\alpha}}} \sum_{x=0}^{p^{N}-1} f(x+3)(-1)^{x+3} q^{(\alpha+\beta)(x+3)} \\
= & \lim _{N \rightarrow \infty} \frac{1}{\left[p^{N}\right]_{-q^{\alpha}}} \sum_{x=0}^{p^{N}-1} f(x)(-1)^{x} q^{(\alpha+\beta) x} \\
& +\lim _{N \rightarrow \infty} \frac{1}{\left[p^{N}\right]_{-q^{\alpha}}}\left(f\left(p^{N}\right)(-1)^{p^{N}} q^{(\alpha+\beta) p^{N}}+f\left(p^{N}+1\right)(-1)^{p^{N}+1} q^{(\alpha+\beta)\left(p^{N}+1\right)}\right. \\
& \left.+f\left(p^{N}+2\right)(-1)^{p^{N}+2} q^{(\alpha+\beta)\left(p^{N}+2\right)}-f(0)+f(1) q^{\alpha+\beta}+f(2) q^{2(\alpha+\beta)}\right) \\
= & I_{q^{\alpha}, \beta}(f)-[2]_{q^{\alpha}} \sum_{x=0}^{2}(-1)^{x+1} f(x) q^{(\alpha+\beta) x} .
\end{aligned}
$$

Continuing this process, we obtain the following theorem.

Theorem 2.3. For each positive integer $n$, if we put $f(x+n)=f_{n}$, then

$$
q^{n(\alpha+\beta)} I_{q^{\alpha}, \beta}\left(f_{n}\right)+(-1)^{n-1} I_{q^{\alpha}, \beta}(f)=[2]_{q^{\alpha}} \sum_{x=0}^{n-1} f(x)(-1)^{n-1-x} q^{(\alpha+\beta) x} .
$$

In view point of (1.1) and (1.2), we note that

$$
\sum_{n=0}^{\infty} \int_{\mathbb{Z}_{p}} q^{\beta y}(x+y)_{n, \lambda} d \mu_{-q^{\alpha}}(y) \frac{t^{n}}{n !}=\int_{\mathbb{Z}_{p}} q^{\beta y}(1+\lambda t)^{\frac{x+y}{\lambda}} d \mu_{-q^{\alpha}}(y),
$$

where $(x)_{n, a}=x(x-a)(x-2 a) \cdots(x-a(n-1)), \lambda, t \in \mathbb{C}_{p}$ with $|\lambda t|_{p}<p^{-\frac{1}{1-p}}$. By the Theorem 2.3, we have

$$
\int_{\mathbb{Z}_{\mathfrak{p}}} q^{\beta y}(1+\lambda t)^{\frac{x+y}{\lambda}} d \mu_{-q^{\alpha}}(y)=\frac{[2]_{q^{\alpha}}}{q^{\alpha+2 \beta}(1+\lambda t)^{\frac{1}{\lambda}}+1}(1+\lambda t)^{\frac{x}{\lambda}} .
$$

By (2.1), we can define the degenerate q-Euler polynomials with weight $\alpha$ as follows.

Definition 2.4. For each positive integer $n$, the degenerate q-Euler polynomials with weight $\alpha$ are defined by the generating function to be

$$
\sum_{n=0}^{\infty} \mathcal{E}_{q^{\alpha}, n}(x \mid \beta) \frac{t^{n}}{n !}=\frac{[2]_{q^{\alpha}}}{q^{\alpha+2 \beta}(1+\lambda t)^{\frac{1}{\lambda}}+1}(1+\lambda t)^{\frac{x}{\lambda}}
$$

In the special case $x=0, \mathcal{E}_{\mathbf{q}^{\alpha}, \mathrm{n}}(0 \mid \beta):=\mathcal{E}_{\mathbf{q}^{\alpha}, \mathrm{n}}(\beta)$ are called the degenerate $\mathrm{q}$-Euler numbers with weight $\alpha$. 
By (2.1), we get

$$
\begin{aligned}
\int_{\mathbb{Z}_{p}} q^{\beta y}(1+\lambda t)^{\frac{x+y}{\lambda}} d \mu_{-q^{\alpha}}(y) & =\int_{\mathbb{Z}_{p}} q^{\beta y} \sum_{n=0}^{\infty}\left(\begin{array}{c}
\frac{x+y}{\lambda} \\
n
\end{array}\right)(\lambda t)^{n} d \mu_{-q^{\alpha}}(y) \\
& =\sum_{n=0}^{\infty} \int_{\mathbb{Z}_{p}} q^{\beta y}(x+y)_{n, \lambda} d \mu_{-q^{\alpha}}(y) \frac{t^{n}}{n !},
\end{aligned}
$$

and thus by the Definition 2.4 and (2.3), we get the Witt's formula for degenerate q-Euler polynomials with weight $\alpha$.

Corollary 2.5. For each nonnegative integer $n$,

$$
\mathcal{E}_{q^{\alpha}, n}(x \mid \beta)=\int_{\mathbb{Z}_{p}} q^{\beta y}(x+y)_{n, \lambda} d \mu_{-q^{\alpha}}(y) .
$$

Note that by (1.4) and the Corollary 2.5, we get

$$
\begin{aligned}
\mathcal{E}_{q^{\alpha}, n}(x \mid \beta) & =\int_{\mathbb{Z}_{p}} q^{\beta y}(x+y)_{n, \lambda} d \mu_{-q^{\alpha}}(y) \\
& =\frac{\lambda^{n}}{n !} \int_{\mathbb{Z}_{p}} q^{\beta y}\left(\frac{x+y}{\lambda}\right)_{n} d \mu_{-q^{\alpha}}(y)=\sum_{l=0}^{n} S_{1}(n, l) \frac{\lambda^{n-l}}{n !} \int_{\mathbb{Z}_{p}} q^{\beta y}(x+y)^{l} d \mu_{-q^{\alpha}}(y) .
\end{aligned}
$$

If $\alpha=1$ and $\beta=0$, then $\int_{\mathbb{Z}_{p}} q^{\beta y}(x+y)^{l} d \mu_{-q^{\alpha}}(y)$ is the $q$-Euler polynomial, and thus

$$
E_{q^{\alpha}, n}(x \mid \beta)=\int_{\mathbb{Z}_{p}} q^{\beta y}(x+y)^{n} d \mu_{-q^{\alpha}}(y)
$$

are called the $q$-Euler polynomials with weight $\alpha$. Hence we obtain the relationship among $\mathcal{E}_{q^{\alpha}, n}(x \mid \beta)$, $E_{q^{\alpha}, n}(x \mid \beta)$, and $S_{1}(n, l)$ as follow.

Corollary 2.6. For each $\mathrm{n} \in \mathbb{N} \cup\{0\}$,

$$
\mathcal{E}_{q^{\alpha}, n}(x \mid \beta)=\sum_{l=0}^{n} \frac{S_{1}(n, l)}{n !} \lambda^{n-l} E_{q^{\alpha}, l}(x \mid \beta) .
$$

From Definition 2.4, we can derive the following equations:

$$
\begin{aligned}
\sum_{n=0}^{\infty} \varepsilon_{q^{\alpha}, n}(x \mid \beta) \frac{t^{n}}{n !} & =\frac{[2]_{q^{\alpha}}}{q^{\alpha+2 \beta}(1+\lambda t)^{\frac{1}{\lambda}}+1}(1+\lambda t)^{\frac{x}{\lambda}} \\
& =\left(\sum_{n=0}^{\infty} \varepsilon_{q^{\alpha}, n}(\beta) \frac{t^{n}}{n !}\right)\left(\sum_{n=0}^{\infty}\left(\begin{array}{c}
\frac{x}{\lambda} \\
n
\end{array}\right)(\lambda t)^{n}\right) \\
& =\sum_{n=0}^{\infty}\left(\sum_{m=0}^{n}\left(\begin{array}{c}
n \\
m
\end{array}\right) \varepsilon_{q^{\alpha}, m}(\beta)(x)_{n-m, \lambda}\right) \frac{t^{n}}{n !} .
\end{aligned}
$$

Hence, we obtain the following corollary.

Corollary 2.7. For each nonnegative integer $n$,

$$
\mathcal{E}_{q^{\alpha}, n}(x \mid \beta)=\sum_{m=0}^{n}\left(\begin{array}{l}
n \\
m
\end{array}\right) \varepsilon_{q^{\alpha}, m}(\beta)(x)_{n-m, \lambda} .
$$


By replacing $t$ by $\frac{1}{\lambda}\left(e^{t}-1\right)$ in (2.2), we get

$$
\begin{aligned}
\frac{[2]_{q^{\alpha}}}{q^{\alpha+2 \beta} e^{\frac{t}{\lambda}}+1} e^{\frac{x t}{\lambda}} & =\sum_{n=0}^{\infty} \varepsilon_{q^{\alpha}, n}(x \mid \beta) \frac{1}{n !}\left(\frac{1}{\lambda}\left(e^{t}-1\right)\right)^{n} \\
& =\sum_{n=0}^{\infty} \varepsilon_{q^{\alpha}, n}(x \mid \beta) \frac{1}{n !} \lambda^{-n} n ! \sum_{l=n}^{\infty} S_{2}(l, n) \frac{t^{l}}{l !} \\
& =\sum_{n=0}^{\infty}\left(\sum_{m=0}^{n} \varepsilon_{q^{\alpha}, m}(x \mid \beta) \lambda^{-m} S_{2}(m, n)\right) \frac{t^{n}}{n !} .
\end{aligned}
$$

Note that by the Theorem 2.3 and (2.4),

$$
\sum_{n=0}^{\infty} E_{q^{\alpha}, n}(x \mid \beta) \frac{t^{n}}{n !}=\int_{\mathbb{Z}_{p}} q^{\beta y} e^{(x+y) t} d \mu_{-q^{\alpha}}(y)=\frac{[2]_{q^{\alpha}}}{q^{\alpha+2 \beta} e^{t}+1} e^{x t} .
$$

From (2.5) and (2.6), we obtain the following theorem.

Theorem 2.8. For each nonnegative integer $n$,

$$
E_{q^{\alpha}, n}(x \mid \beta)=\sum_{m=0}^{n} \varepsilon_{q^{\alpha}, m}(x \mid \beta) \lambda^{n-m} S_{2}(m, n) .
$$

From now on, we consider another degenerate q-Euler polynomials with weight $\alpha$. In viewpoint of the Definition 2.1 and the Corollary 2.5, we define those polynomials as follows.

Definition 2.9. For each nonnegative integer $n$,

$$
\widehat{\mathcal{E}}_{q^{\alpha}, n}(x \mid \beta)=\int_{\mathbb{Z}_{p}} q^{\beta y}(x-y)_{n, \lambda} d \mu_{-q^{\alpha}}(y)
$$

are called the degenerate q-Euler polynomials of the second kind with weight $\alpha$.

By the Definition 2.9, the generating function of the degenerate q-Euler polynomials of the second kind with weight $\alpha$ is as following:

$$
\begin{aligned}
\sum_{n=0}^{\infty} \widehat{\mathcal{E}}_{q^{\alpha}, n}(x \mid \beta) \frac{t^{n}}{n !} & =\sum_{n=0}^{\infty} \int_{\mathbb{Z}_{p}} q^{\beta y}(x-y)_{n, \lambda} d \mu_{-q^{\alpha}}(y) \frac{t^{n}}{n !} \\
& =\int_{\mathbb{Z}_{p}} q^{\beta y}\left(\sum_{n=0}^{\infty}\left(\begin{array}{c}
\frac{x-y}{\lambda} \\
n
\end{array}\right) \frac{(\lambda t)^{n}}{n !}\right) d \mu_{-q^{\alpha}}(y) \\
& =\int_{\mathbb{Z}_{p}} q^{\beta y}(1+\lambda t)^{\frac{x-y}{\lambda}} d \mu_{-q^{\alpha}}(y) \\
& =\frac{[2]_{q^{\alpha}}}{q^{\alpha+2 \beta}+(1+\lambda t)^{\frac{1}{\lambda}}}(1+\lambda t)^{\frac{x+1}{\lambda}}
\end{aligned}
$$

and by (1.4) and Theorem 2.8, we have

$$
\begin{aligned}
\widehat{\mathcal{E}}_{q^{\alpha}, n}(x \mid \beta) & =\int_{\mathbb{Z}_{p}} q^{\beta y}(x-y)_{n, \lambda} d \mu_{-q^{\alpha}}(y) \\
& =\int_{\mathbb{Z}_{p}} q^{\beta y} \lambda^{n}\left(\frac{x-y}{\lambda}\right)_{n} d \mu_{-q^{\alpha}}(y)
\end{aligned}
$$




$$
\begin{aligned}
& =\int_{\mathbb{Z}_{p}} q^{\beta y} \sum_{l=0}^{n} \lambda^{n-l} S_{1}(n, l)(x-y)^{l} d \mu_{-q^{\alpha}}(y) \\
& =\sum_{l=0}^{n} \lambda^{n-l} S_{1}(n, l)(-1)^{l} \int_{\mathbb{Z}_{p}} q^{\beta y}(-x+y)^{l} d \mu_{-q^{\alpha}}(y) \\
& =\sum_{l=0}^{n} S_{1}(n, l)(-1)^{l} \lambda^{n-l} E_{q^{\alpha}, l}(-x) \\
& =\sum_{l=0}^{n} \sum_{m=0}^{l}(-1)^{l} \lambda^{n-l} S_{1}(n, l) S_{2}(m, l) \mathcal{E}_{q^{\alpha}, m}(-x \mid \beta)
\end{aligned}
$$

for each nonnegative integer $n$.

By (2.7) and (2.8), we obtain the following theorem.

Theorem 2.10. For each nonnegative integer $n$,

$$
\begin{aligned}
& \widehat{\mathcal{E}}_{\mathrm{q}^{\alpha}, \mathrm{n}}(x \mid \beta)=\sum_{l=0}^{n} S_{1}(n, l)(-1)^{l} \lambda^{n-l} E_{q^{\alpha}, l}(-x), \\
& \widehat{\mathcal{E}}_{\mathrm{q}^{\alpha}, \mathrm{n}}(x \mid \beta)=\sum_{l=0}^{n} \sum_{m=0}^{l}(-1)^{l} \lambda^{n-l} S_{1}(n, l) S_{2}(m, l) \mathcal{E}_{q^{\alpha}, m}(-x \mid \beta),
\end{aligned}
$$

and the generating function of the q-Changhee polynomials of the second kind with weight $\alpha$ is

$$
\sum_{n=0}^{\infty} \widehat{\mathcal{E}}_{q^{\alpha}, n}(x \mid \beta) \frac{t^{n}}{n !}=\int_{\mathbb{Z}_{p}} q^{\beta y}(1+\lambda t)^{\frac{x-y}{\lambda}} d \mu_{-q^{\alpha}}(y)=\frac{[2]_{q^{\alpha}}}{q^{\alpha+2 \beta}+(1+\lambda t)^{\frac{1}{\lambda}}}(1+\lambda t)^{\frac{x+1}{\lambda}} .
$$

By replacing t by $\frac{1}{\lambda}\left(e^{t}-1\right)$ in (2.9), we have

$$
\begin{aligned}
\frac{[2]_{q^{\alpha}}}{q^{\alpha+2 \beta}+e^{\frac{t}{\lambda}}} e^{\frac{x+1}{\lambda} t} & =\sum_{n=0}^{\infty} \widehat{\mathcal{E}}_{q^{\alpha}, n}(x \mid \beta) \frac{\left(\frac{1}{\lambda}\left(e^{t}-1\right)\right)^{n}}{n !} \\
& =\sum_{n=0}^{\infty} \widehat{\mathcal{E}}_{q^{\alpha}, n}(x \mid \beta) \lambda^{-n} \frac{1}{n !} n ! \sum_{l=n}^{\infty} S_{2}(l, n) \frac{t^{l}}{l !} \\
& =\sum_{n=0}^{\infty}\left(\sum_{m=0}^{n} \widehat{\mathcal{E}}_{q^{\alpha}, m}(x \mid \beta) \lambda^{-m} S_{2}(n, m)\right) \frac{t^{n}}{n !}
\end{aligned}
$$

and

$$
\begin{aligned}
\frac{[2]_{q^{\alpha}}}{q^{\alpha+2 \beta}+e^{\frac{t}{\lambda}}} e^{\frac{x+1}{\lambda} t} & =\frac{[2]_{q^{\alpha}}}{q^{\alpha+2 \beta} e^{-\frac{t}{\lambda}}+1} e^{(-x)\left(-\frac{t}{\lambda}\right)} \\
& =\sum_{n=0}^{\infty} E_{q^{\alpha}, n}(-x \mid \beta) \frac{\left(-\frac{t}{\lambda}\right)^{n}}{n !}=\sum_{n=0}^{\infty} E_{q^{\alpha}, n}(-x \mid \beta)(-\lambda)^{-n} \frac{t^{n}}{n !} .
\end{aligned}
$$

By (2.10) and (2.11), we obtain the following theorem.

Theorem 2.11. For each nonnegative integer $n$, we have

$$
E_{q^{\alpha}, n}(-x \mid \beta)=(-\lambda)^{n} \sum_{m=0}^{n} \widehat{\varepsilon}_{q^{\alpha}, m}(x \mid \beta) \lambda^{-m} S_{2}(n, m) .
$$


By the Definition 2.4 and the Theorem 2.10, we note that

$$
\begin{aligned}
& \sum_{n=0}^{\infty} \widehat{\varepsilon}_{q^{\alpha}, n}(x \beta) \frac{t^{n}}{n !}=\frac{[2]_{q^{\alpha}}}{q^{\alpha+2 \beta}+(1+\lambda t)^{\frac{1}{\lambda}}}(1+\lambda t)^{\frac{x+1}{\lambda}} \\
& =q^{-\alpha-2 \beta} \frac{[2]_{q^{\alpha}}}{q^{-\alpha-2 \beta}(1+\lambda t)^{\frac{1}{\lambda}}+1}(1+\lambda t)^{\frac{x+1}{\lambda}} \\
& =q^{-2 \beta} \frac{[2]_{q^{-\alpha}}}{q^{-\alpha-2 \beta}(1+\lambda t)^{\frac{1}{\lambda}}+1}(1+\lambda t)^{\frac{x+1}{\lambda}} \\
& =\sum_{n=0}^{\infty} q^{-2 \beta} \varepsilon_{q^{-\alpha}, n}(x+1 \mid-\beta) \frac{t^{n}}{n !} \text {. }
\end{aligned}
$$

In addition, by the Corollary 2.5 and the Definition 2.9,

$$
\begin{aligned}
\frac{(-1)^{n} \mathcal{E}_{q^{\alpha}, n}(x \mid \beta)}{n !} & =\frac{(-1)^{n}}{n !} \int_{\mathbb{Z}_{p}} q^{\beta y}(x+y)_{n, \lambda} d \mu_{-q^{\alpha}}(y) \\
& =\lambda^{n} \int_{\mathbb{Z}_{p}} q^{\beta}\left(\begin{array}{c}
n-1-\frac{x+y}{\lambda} \\
n
\end{array}\right) d \mu_{-q^{\alpha}}(y) \\
& =\lambda^{n} \sum_{k=0}^{n-1}\left(\begin{array}{c}
n-1 \\
n-k
\end{array}\right) \int_{\mathbb{Z}_{p}} q^{\beta y}\left(\begin{array}{c}
\frac{-x-y}{\lambda} \\
k
\end{array}\right) d \mu_{-q^{\alpha}}(y) \\
& =\sum_{k=0}^{n-1} \lambda^{n-k}\left(\begin{array}{c}
n-1 \\
n-k
\end{array}\right) \int_{\mathbb{Z}_{p}} q^{\beta y} \frac{(-x-y)_{n, k}}{k !} d \mu_{-q^{\alpha}}(y) \\
& =\sum_{k=1}^{n-1} \lambda^{n-k}\left(\begin{array}{c}
n-1 \\
k-1
\end{array}\right) \frac{\widehat{\mathcal{E}}_{q^{\alpha}, k}(-x \mid \beta)}{k !}
\end{aligned}
$$

and

$$
\begin{aligned}
\frac{(-1)^{n} \widehat{\mathcal{E}}_{q^{\alpha}, n}(x \mid \beta)}{n !} & =\frac{(-1)^{n}}{n !} \int_{\mathbb{Z}_{p}} q^{\beta y}(x-y)_{n, \lambda} d \mu_{-q^{\alpha}}(y) \\
& =\lambda^{n} \int_{\mathbb{Z}_{p}} q^{\beta}\left(\begin{array}{c}
n-1-\frac{x-y}{\lambda} \\
n
\end{array}\right) d \mu_{-q^{\alpha}}(y) \\
& =\lambda^{n} \sum_{k=0}^{n-1}\left(\begin{array}{c}
n-1 \\
n-k
\end{array}\right) \int_{\mathbb{Z}_{p}} q^{\beta y}\left(\begin{array}{c}
\frac{-x+y}{\lambda} \\
k
\end{array}\right) d \mu_{-q^{\alpha}}(y) \\
& =\sum_{k=0}^{n-1} \lambda^{n-k}\left(\begin{array}{c}
n-1 \\
n-k
\end{array}\right) \int_{\mathbb{Z}_{p}} q^{\beta y} \frac{(-x+y)_{k, \lambda}}{k !} d \mu_{-q^{\alpha}}(y) \\
& =\sum_{k=1}^{n-1} \lambda^{n-k}\left(\begin{array}{c}
n-1 \\
k-1
\end{array}\right) \frac{\mathcal{E}_{q^{\alpha}, k}(-x \mid \beta)}{k !} .
\end{aligned}
$$

Thus, by (2.12), (2.13), and (2.14), we obtain the following theorem.

Theorem 2.12. For each nonnegative integer $n$, we have

$$
\begin{aligned}
& \widehat{\mathcal{E}}_{\mathrm{q}^{\alpha}, \mathrm{n}}(x \beta)=\mathrm{q}^{-2 \beta} \mathcal{E}_{\mathrm{q}^{-\alpha}, \mathrm{n}}(x+1 \mid-\beta), \\
& \mathcal{E}_{\mathrm{q}^{\alpha}, \mathrm{n}}(x \mid \beta)=(-1)_{n} \sum_{k=1}^{n-1} \lambda^{n-k}\left(\begin{array}{c}
n-1 \\
k-1
\end{array}\right) \frac{\widehat{\mathcal{E}}_{\mathrm{q}^{\alpha}, \mathrm{k}}(-x \mid \beta)}{k !},
\end{aligned}
$$


and

$$
\widehat{\mathcal{E}}_{\mathrm{q}^{\alpha}, \mathrm{n}}(x \mid \beta)=(-1)_{\mathrm{n}} \sum_{k=1}^{n-1} \lambda^{n-k}\left(\begin{array}{c}
n-1 \\
k-1
\end{array}\right) \frac{\mathcal{E}_{\mathrm{q}^{\alpha}, \mathrm{k}}(-x \mid \beta)}{k !} .
$$

\section{Acknowledgment}

The authors would like to thank the referees for many valuable and detailed comments. This work was supported by Daegu University Undergraduate Research Program, 2019.

\section{References}

[1] A. Adelberg, A finite difference approach to degenerate Bernoulli and Stirling polynomials, Discrete Math., 140 (1995), 1-21. 1

[2] G. E. Andrews, R. Askey, R. Roy, Special Functions, Cambridge University Press, Cambridge, (1999). 1

[3] S. Araci, E. Ağyüz, M. Acikgoz, On a q-analog of some numbers and polynomials, J. Inequal. Appl., 2015 (2015), 9 pages. 1

[4] S. Araci, U. Duran, M. Acikgoz, On weighted q-Daehee polynomials with their applications, Indag. Math. (N.S.), 30 (2019), 365-374. 1, 1.2

[5] L. Carlitz, q-Bernoulli and Eulerian numbers, Trans. Amer. Math. Soc., 76 (1954), 332-350. 1.2, 1

[6] L. Carlitz, Degenerate Stirling, Bernoulli and Eulerian numbers, Utilitas Math., 15 (1979), 51-88. 1, 1.1, 1, 1, 1.7

[7] C. Cesarano, Operational methods and new identities for Hermit polynomials, Math. Model. Nat. Phenom., 12 (2017), 44-50. 1

[8] L. Comtet, Advanced Combinatorics, D. Reidel Publishing Co., Dordrecht, (1974). 1, 1.7, 1

[9] D. V. Dolgy, G. W. Jang, H.-I. Kwon, T. Kim, A note on Carlitzs type q-Changhee numbers and polynomials, Adv. Stud. Contemp. Math., 27 (2017), 451-459. 1

[10] S. Gaboury, R. Tremblay, B.-J. Fugere, Some explicit formulas for certain new classes of Bernoulli, Euler and Genocchi polynomials, Proc. Jangjeon Math. Soc., 17 (2014), 115-123. 1, 1.1

[11] T. Kim, On q-analogue of the p-adic log gamma functions and related integral, J. Number Theory, 76 (1999), $320-329$. 1.1

[12] T. Kim, q-Volkenborn integration, Russ. J. Math. Phys., 9 (2002), 288-299. 1, 1.1, 1, 2.2

[13] T. Kim, q-Euler numbers and polynomials associated with p-adic q-integral, J. Nonlinear Math. Phys., 14 (2007), $15-27$.

[14] T. Kim, Some identities on the q-Euler polynomials of higher order and q-Stirling numbers by the fermionic p-adic integral on $\mathbb{Z}_{\mathrm{p}}$, Russ. J. Math. Phys., 16 (2009), 484-491. 1, 1.7

[15] T. Kim, A study on the q-Euler numbers and the fermionic q-integrals of the product of several type q-Bernstein polynomials on $\mathbb{Z}_{\mathrm{p}}$, Adv. Stud. Contemp. Math. (Kyungshang), 23 (2013), 5-11.

[16] B. M. Kim, L.-C. Jang, W. Kim, H.-I. Kwon, On Carlitz's Type Modified Degenerate Changhee Polynomials and Numbers, Discrete Dyn. Nat. Soc., 2018 (2018), 5 pages. 1.2, 1

[17] D. S. Kim, T. Kim, Three variable symmetric identities involving Carlitz-type q-Euler polynomials, Math. Sci. (Springer), 8 (2014), 147-152. 1.2, 1

[18] D. S. Kim, T. Kim, Some p-adic integrals on $\mathbb{Z}_{p}$ associated with trigonometric Functions, Russ. J. Math. Phys., 25 (2018), 300-308.

[19] T. Kim, D. S. Kim, D. V. Dolgy, Degenerate q-Euler polynomials, Adv. Difference Equ., 2015 (2015), 11 pages.

[20] T. Kim, H.-I. Kwon, J. J. Seo, Degenerate q-Changhee polynomials, J. Nonlinear Sci. Appl., 9 (2016), 2389-2393. 1

[21] T. Kim, J. J. Seo, Revisit nonlinear differential equations arising from the generating functions of degenerate Bernoulli numbers, Adv. Stud. Contemp. Math., 26 (2016), 401-406. 1

[22] J. K. Kwon, J.-W. Park, On modified degenerate Changhee polynomials and numbers, J. Nonlinear Sci. Appl., 9 (2016), 6294-6301. 1

[23] C. Liu, Wuyungaowa, Application of Probabilistic Method on Daehee Sequences, Eur. J. Pure Appl. Math., 11 (2018), 69-78. 1

[24] M. Marin, Weak solutions in elasticity of dipolar porous materials, Math. Probl. Eng., 2008 (2008), 8 pages. 1

[25] H.-K. Pak, J. H. Jeong, D.-J. Kang, S.-H. Rim, Changhee-Genocchi numbers and their applications, Ars Combin., 136 (2018), 153-159.

[26] C. S. Ryoo, On the extended q-Euler numbers and polynomials with weak weight $\alpha$, Far East J. Math. Sci. (FJMS), 70 (2012), 365-373. 1

[27] A. Sharma, q-Bernoulli and Euler numbers of higher order, Duke Math. J., 25 (1958), 343-353.

[28] Y. Simsek, Identities on the Changhee numbers and Apostol-type Daehee polynomials, Adv. Stud. Contemp. Math. (Kyungshang), 27 (2017), 199-212. 1.2

[29] H. M. Srivastava, Some generalizations and basic (or q-) extensions of the Bernoulli, Euler and Genocchi polynomials, Appl. Math. Inf. Sci., 5 (2011), 390-444. 1, 1.2, 1 